\title{
Géneros, autoría y audiencia en la creación de una revista estudiantil digital
}

\section{Genres, Authorship and Audience in the Process of Creating a Digital Student Magazine}

\author{
DOI: http://dx.doi.org/10.17981/cultedusoc.11.1.2020.09
}

Recibido: 01/11/2019 Aceptado: 19/02/2020. Publicado: 20/03/2020

\section{Sol Colmenares Rodríguez}

Universidad del Valle. Cali (Colombia)

sol.colmenares.rodriguez@correounivalle.edu.co

Para citar este artículo:

Colmenares, S. (2020). Géneros, autoría y audiencia en la creación de una revista estudiantil digital. Cultura, Educación y Sociedad, 11(1). 127-138. DOI: http://dx.doi.org/10.17981/cultedusoc.11.1.2020.09

\section{Resumen}

Las revistas creadas por estudiantes son proyectos pedagógicos que ofrecen la oportunidad de abordar la escritura de distintos géneros con una audiencia concreta, una práctica fundamental para autores en potencia. En este artículo se presentan resultados parciales de la investigación Ritos de iniciación científica: el proceso de creación de una revista estudiantil digital, con el fin de analizar cómo un grupo de estudiantes de una licenciatura en lenguas extranjeras, asume la tarea de crear contenidos para una revista estudiantil digital. En el marco metodológico de la sistematización de experiencias, se analiza el corpus compuesto de textos escritos por estudiantes y los datos obtenidos a través de encuestas, fichas de observación y entrevistas para responder a los siguientes interrogantes: ¿Qué géneros discursivos reconocen los estudiantes como propios de una revista digital dirigida a aprendices de lenguas extranjeras? y ¿qué géneros consideran los docentes deben incorporarse en una revista digital estudiantil? Los resultados destacan al ensayo como el género más común encontrado en el corpus e igualmente resaltan instructivos, consejos y relatos biográficos de notorio significado en las experiencias identificadas. Se concluye en la importancia de la academia para la conformación de experiencias personales, relatos autobiográficos e historias de vida como referentes legítimos, no solo para la búsqueda de temas de escritura, sino también con relación al uso efectivo de estrategias retóricas y para la construcción de autoría.

Palabras clave: Escritura; autor; audiencia; revista; pedagogía.

\section{Abstract}

Student-created journals are pedagogical projects that offer the opportunity to approach writing in different genres with a specific audience, a fundamental practice for potential authors. This article presents partial results of the research Rites of Scientific Initiation: the process of creating a digital student journal, in order to analyze how a group of students from a foreign language degree take on the task of creating content for a digital student journal. Within the methodological framework of experience systematisation, the corpus composed of texts written by students and data obtained through surveys, observation sheets and interviews is analysed to answer the following questions: What discursive genres do students recognise as belonging to a digital journal aimed at foreign language learners? and What genres do teachers consider should be incorporated into a digital student journal? The results highlight the essay as the most common genre found in the corpus and equally highlight instructional, advice and biographical accounts of notable significance in the experiences identified. We conclude with the importance of the academy for the formation of personal experiences, autobiographical stories and life stories as legitimate references, not only for the search of writing topics, but also in relation to the effective use of rhetorical strategies and for the construction of authorship.

Keywords: Writing; author; audience; magazine; pedagogy. 


\section{INTRODUCCIÓN}

Los seres humanos tienen la necesidad de sentir que sus historias importan. "Si el cuerpo se alimenta de comida, el alma se alimenta de agencia y significatividad" (Gee, 2013, p. 10). Si se extrapola esta idea al campo de la didáctica de la escritura en el ámbito universitario, es posible afirmar que los temas que interesan a los estudiantes, que les resultan familiares y sobre los cuales quieren escribir pueden convertirse en poderosos dispositivos para el desarrollo de prácticas de escritura relevantes, no solo para su profesión, sino también para otras dimensiones de su identidad tan importantes como la profesional.

Un ejemplo que ilustra la afirmación anterior puede encontrarse en el proyecto dirigido por las investigadoras Elizabeth Daley y Stephanie Barish, en el cual "estudiantes con una resistencia abierta a escribir en la escuela expresaron sus perspectivas sobre la violencia armada, con la cual estaban muy familiarizados. Inspirados en sus propios remixes de videos, los estudiantes 'se tropezaron con el hecho [de que tenían] que explicar esto ... y realmente [necesitaban] escribir algo" (Lankshear y Knobel, 2008, p. 9). Ello garantiza el no abandono de la lucha que supone la escritur y la motivación continua; es importante sostener la necesidad auténtica de escribir, el deseo genuino de quien escribe para comunicar sus ideas de la mejor manera posible, haciendo un uso creativo de los recursos expresivos a su disposición.

Lankshear y Knobel (2008) proponen una redefinición de la cultura académica de manera que involucre "participación profesional, técnica, administrativa, cívica y de otros tipos en modos de hacer y ser que sean relevantes para las trayectorias de vida posteriores a la escuela" (p. 12). Estos modos de ser y hacer no son exclusivos de la vida futura de los estudiantes, sino que hacen parte de su presente y están atravesados por identidades digitales. Es aquí donde se vuelve vital la labor docente, en especial en el sector público, ya que tal como advierten los autores: "la brecha en términos de literacidad digital que existe entre estudiantes pobres y estudiantes ricos sigue profundizando la ya tradicional brecha existente en las prácticas de lectura y escritura” (Lankshear y Knobel, 2008, p. 13 ).

Contribuir a minimizar esta brecha es incluso un asunto de justicia social en el que el rol de los profesores debe ser "preparar a los estudiantes menos favorecidos para que tengan representación y participación completa en sus sociedades” (Miller \& Kelly, 2016, p. 274). Las competencias que el mundo tecnológico actual exige de los jóvenes "supone la adquisición sistemática de una nueva literacidad" (Hyland, 2006, p. 35). Contrario a lo que comúnmente se encuentra en el pensamiento docente, el solo hecho de ser joven no garantiza ser competente en el uso de la tecnología o tener altos niveles de literacidad digital. Es por esta razón que resulta necesario diseñar propuestas pedagógicas para la creación y comunicación de contenidos: "Crear contenido digital y comunicarlo es un proceso intelectual asociado a las prácticas de literacidad digital” (Medlock, Spires y Kerkhoff, 2017, p. 2235). Por tanto es necesario construir escenarios en los cuales los estudiantes sean creadores de sentido y no solo consumidores, se infiere una acción descolonizadora fundamental para la creación de conocimiento contextualizado en lo local.

Además de lo anterior, crear y comunicar contenido digital significa construirse como autor "creando mensajes mediáticos del mundo real para audiencias auténticas" (Hobbs y 
Smith, 2018, p. 3), audiencias que pueden reaccionar, contra-argumentar, e incluso insultar y matonear. El concepto de audiencia tan escurridizo en el pasado, se ha convertido ahora en una entidad más tangible que puede ser pensada en términos de "intereses, conocimiento, valores y expresiones retóricas" y que es construida "por escritores expertos quienes entienden la importancia de estas características y las abordan en todas las fases de composición" (Zainuddin y Moore, 2003, párr. 4). "Los lectores deben ser atraídos, influenciados y persuadidos (...). Para reclamar el derecho de ser escuchado y que su trabajo sea tomado seriamente, los escritores deben mostrarse como conocedores de la disciplina y esta competencia es, por lo menos en parte, alcanzada a través de un diálogo entre el escritor y el lector" (Hyland, 2006, p. 36). Es esta construcción de la audiencia lo que garantiza la pertenencia a una comunidad discursiva que comparte saberes y prácticas.

Tener conciencia de la audiencia tiene también implicaciones epistemológicas: "Problematizar lo escrito desde el enfoque atribuido al destinatario implica cuestionar el conocimiento disponible" (Carlino, 2005, p. 28); una tarea imprescindible en los ejercicios de escritura persuasiva. Por ejemplo. Blanco y Mosquera (2019), en su trabajo "El lenguaje valorativo en artículos de investigación sobre interculturalidad en la enseñanza de lenguas» concluyen que "cuando hay una mayor conciencia de la audiencia, se pueden construir argumentos más fuertes" (p. 15). Asimismo, para Hobbs y Smith (2018) "cuando los estudiantes crean medios, deben considerar la compleja relación de poder que existe entre ellos mismos como autores, el sujeto que ellos representan en su trabajo creativo y la audiencia que recibe el mensaje" (p. 4). Una experiencia que solo es posible cuando los textos escritos tienen la oportunidad de circular y llegar a lectores diferentes al docente. Al respecto, Urra y Pérez (2015) destacan la importancia de la divulgación de la información a través de los medios más idóneos en correspondencia con el tipo de lector.

Autoría y audiencia se superponen así como en una cinta de Moebius en un movimiento constante de ida y vuelta, más aún cuando -como en el caso de la investigación que se reporta- los estudiantes crean contenidos para sus pares. Ser audiencia, sin embargo, pareciera no implicar tantas "responsabilidades" como la autoría.

Para un discurso el hecho de tener un nombre de autor, el hecho de poder decir 'esto fue escrito por Fulano de Tal' indica que dicho discurso no es una palabra cotidiana, indiferente, una palabra que se va, que flota y pasa, una palabra que puede consumirse inmediatamente sino que se trata de una palabra que debe recibirse de cierto modo y que debe recibir, en una cultura dada, un cierto estatuto. (Foucault, 1969, p. 60).

En otras palabras, "un autor es una voz, un eco, una consecuencia" (...) tiene "repercusiones" y es interesante que la noción de afectar el mundo tenga que ver con el gesto de 'percutir', de 'percusión', de crear un ritmo determinado" (Flórez, 2018).

Para otros, ser autor significa también responder por aquello que se dice, incluso si la consecuencia es el castigo:

Hacer públicos los textos producidos significa poner en circulación al autor como sujeto que se expone en contextos sociales concretos: se expone a la crítica, a la censura, pero así mismo puede obtener el reconocimiento y la admiración de los otros (Carvajal, 2008, pp. 213-214). 
En este mismo sentido, Ana Maria Netto Machado enuncia el asumir públicamente lo escrito como condición principal de la autoría, de manera que lo dicho "pasa a trascender la esfera individual y personal para alcanzar una dimensión colectiva, operando la inserción de lo singular y subjetivo en el espacio público" (Netto, 2007, p. 171), una práctica que se diferencia de la escritura para el profesor o la escritura personal.

De los planteamientos hasta aquí enunciados, es posible inferir que para guiar a los estudiantes en la construcción de autoría y de audiencia, es necesario trabajar sistemáticamente en el reconocimiento de los géneros discursivos como:

1. "Conjuntos de enunciados relativamente estables, que tienen su concepción patrón del destinatario la cual determina su composición” (Bajtín, 1982, p. 321).

2. Herramientas de cognición en el sentido propuesto por Bazerman (2009): "los géneros identifican un problema para que el escritor en desarrollo trabaje en él y proveen la forma de la solución que el escritor busca” (p. 136).

3. Herramientas para "construir nuevos significados en la academia" (Lillis, 2003, p. 205).

4. "Llaves para entender cómo participar en las acciones de una comunidad" (Miller, 1994, p. 67).

Una comprensión tal de los géneros discursivos no se da de manera intuitiva; por el contrario, se alcanza a través de prácticas pedagógicas diseñadas específicamente para este fin. Enseñar a los estudiantes a "leer como escritores, explorando nuevos textos y analizándolos con los ojos de aquellos que construyen esos textos" (Gainer citado por Hobbs y Smith, 2018, p. 7) hace parte de la formación de identidades expertas en tecnología, fundamentales para ejercer agencia en muchas de las prácticas de literacidad contemporáneas. En el seno de esta formación es imprescindible tener en cuenta que los "estudiantes traen diversas literacidades" y es función del docente "mapearlas con aquellas que su aprendizaje requiere" (Mannion, Ivanic \& Literacies for Learning in Further Education-LfLFE, 2007, p. 1). De esta manera, se construyen experiencias significativas que permiten establecer conexiones entre la realidad del contexto y las prácticas que se promueven en el espacio académico.

En este sentido, las revistas digitales estudiantiles son proyectos pedagógicos ideales, ya que ofrecen oportunidades para "el aprendizaje de múltiples habilidades de escritura en contexto" y para el "desarrollo de una comunidad de aprendizaje de la escritura" (Sheedlo, 1998, p. 48). Por tratarse de un medio de comunicación auténtico, en la creación de una revista los estudiantes pueden experimentar con distintos géneros precisando una audiencia concreta y deben llevar a cabo distintas tareas (escritura, edición, corrección de estilo, diseño, etc.) para la consecución de un objetivo en común.

\section{MetodoloGía}

Usada en diferentes campos del conocimiento como la economía rural hasta la investigación en artes, la sistematización de experiencias "es una metodología que facilita la descripción, la reflexión, el análisis y la documentación, de manera continua y participativa de procesos 
y resultados de un proyecto" (Selener, Purdy y Zapata, 1996, p. 7) y permite "explicitar, organizar y, por tanto, hacer comunicables, los saberes adquiridos en la experiencia" (Barnechea y Morgan, 2010, p. 101). Y también es "una reconstrucción de lo sucedido y un ordenamiento de los distintos elementos objetivos y subjetivos que han intervenido en el proceso, para comprenderlo, interpretarlo y así aprender de nuestra propia práctica" (Jara, 2001, p. 2).

Haber participado en la experiencia es otro aspecto fundamental de la sistematización, que permite "objetivar lo vivido explicitando y mirando críticamente lo que hacemos". (Jara, 2001, p. 5). De ahí que la investigadora asuma en este caso el rol de observadorparticipante, como agente que registra el proceso de creación con una intención premeditada, objetivos concretos y la convicción de la riqueza que la experiencia podría generar en el ámbito pedagógico. Esta metodología tiene como principio el aprendizaje colectivo basado en la experiencia y la observación y propende por la reflexión teórica con conocimientos surgidos directamente de las experiencias.

Para efectos de este artículo, se presentan los hallazgos del análisis de dos fuentes: el corpus constituido por 37 textos escritos por estudiantes para una revista digital estudiantil y siete entrevistas semiestructuradas realizadas con docentes del programa para el cual se propone la creación de la revista.

Los textos han sido escritos por estudiantes de la Licenciatura en Lenguas Extranjeras de la Universidad del Valle (Colombia) en los cursos: Tipologías discursivas escritas en inglés, gramática del inglés y taller avanzado de inglés durante los años 2017 y 2018. Estos cursos son tomados por estudiantes que se encuentran entre quinto y séptimo semestre del programa. En todos los casos, la escritura de los textos se hace en el marco de una experiencia pedagógica propuesta por la docente en el programa del curso e incluida en la evaluación. La consigna es escribir un texto (en inglés) para la revista digital La Langosta Magazine de entre 300 y 600 palabras. El género lo escoge cada estudiante, con ayuda de la investigadora/docente, dependiendo de la temática del escrito y la intención comunicativa. Se hace énfasis en que los estudiantes seleccionen temas de su interés, ya sean académicos o no. De igual manera se les pide escribir teniendo en cuenta que la audiencia principal serían sus pares, es decir, otros estudiantes de la licenciatura. Antes de llevar a cabo el ejercicio de escritura los estudiantes revisaron publicaciones de revistas digitales de otras universidades (nacionales e internacionales) y las presentaron al grupo.

Los propósitos de analizar el corpus fueron: identificar géneros discursivos donde se inscriben los textos compilados y rastrear en ellos propósitos comunicativos, temas explorados, tratamiento de la audiencia, patrones de organización, mecanismos retóricos y rasgos estilísticos (Miller \& Kelly, 2016). Una lectura atenta y detallada del corpus en busca de patrones pero también de peculiaridades f el ejercicio principal para nutrir la matriz de análisis. Resistir el deseo de agrupar aquello que parece semejante y optar por agrupar lo diferente, por yuxtaponer cosas que a primera vista parecen no semejarse, (como en el mestizaje, siguiendo el hilo que tiende Flórez en su texto breve Currículo Oculto) es otra acción deliberada que atraviesa la metodología (Flórez, 2018).

La estructura de algunos de los textos analizados facilita la tarea de clasificación. Es el caso de la reseña, la más transparente del corpus, ya que el patrón organizativo descripción- 
narración-opinión es el más común en este tipo de texto. El más borroso resulta ser el de los instructivos y consejos; si bien es posible identificar el análisis de un proceso como el mecanismo retórico dominante en común, las diferencias en los usos de la mitigación, la relevancia o no de las secuencias y el grado de autoridad que la voz del escritor proyecta, evidencian una heterogeneidad en los artículos agrupados en esta categoría. La lectura de Polyzou ilumina la toma de decisiones a la hora de escoger qué textos incluir. Es así que se opta usar como criterio el propósito comunicativo orientado a "proveer consejos a los lectores", se debe identificar una "estructura subyacente de problema-solución" y la presencia de "actos de habla directivos" (Polyzou, 2008, p. 115). Los otros géneros usados como categorías para la clasificación de los textos del corpus fueron: el ensayo, el relato autobiográfico, el texto literario y el reporte de investigación.

Con el análisis de las entrevistas semiestructuradas realizadas, se busca identificar qué géneros esperan encontrar los docentes en una publicación diseñada por y dirigida a estudiantes de una licenciatura en lenguas extranjeras para, posteriormente, contrastar sus expectativas con las producciones estudiantiles analizadas. La duración promedio de las entrevistas es de 30 minutos. En todos los casos se plantea como una interacción amigable entre colegas en la que más que preguntas, se plantearon ejes temáticos tales como la relevancia, identidad, políticas editoriales, roles, referentes y financiación de una revista estudiantil digital para el programa de licenciatura en lenguas extranjeras.

\section{Resultados}

El género más común encontrado en el corpus es el ensayo. Le siguen en número los instructivos y consejos. En tercer lugar se encuentran los relatos autobiográficos. A continuación están las reseñas y por último, con apariciones excepcionales, un reporte de investigación y tres textos literarios.

En los ensayos se evidencia la intención de persuadir a la audiencia, por ejemplo, de lo peligroso que resulta ver a los migrantes como "ellos", por oposición a "nosotros" a propósito de la migración venezolana en Colombia (Them), de la importancia de la memoria colectiva para ejercer una ciudadanía responsable (The importance of collective memory) o de la urgencia de hablar sobre el acoso sexual que experimentan las niñas desde muy temprana edad (We have all been there). Hay un estudiante que plantea una reflexión -a partir de su experiencia como docente de inglés en un instituto- (The $X$ Institute) sobre los distintos estilos de aprendizaje y exhorta a sus lectores para que integren todas las habilidades comunicativas en sus metodologías de enseñanza. En todos estos textos, además de la persuasión, se encuentran también mecanismos retóricos como la definición y la narración.

En la categoría de instructivos se ubican un compendio de recetas saludables (Healthy Recipes in a Hurry) y un artículo sobre rutinas de ejercicios para mantenerse en forma (Don't stress out, work out). Estos textos están organizados de manera secuencial (primero, después, finalmente) y tienen un estilo ágil, directo y sencillo. En cuanto al tratamiento de la audiencia se caracterizan por abordarla de manera clara y contextualizada: las recetas están pensadas para estudiantes con poco tiempo, bajo presupuesto y necesidades 
nutricionales específicas. Asimismo, las rutinas de ejercicios están diseñadas para realizarse dentro del campus universitario, en sesiones de 15 minutos y sin necesidad de equipo o ropa especial.

En este mismo grupo se incluyen los textos en los que se ofrecen consejos y que comparten con los instructivos el mecanismo retórico problema-solución. En uno, se dan recomendaciones para ayudar a una persona con depresión (How to Help Someone with Depression). En otro, se enumeran recomendaciones para mejorar las relaciones personales (Love yourself). En estos escritos se usan preguntas como estrategias para plantear el problema, y aunque se usan verbos en modo imperativo, se mitiga el discurso tratando a la audiencia como una igual. A diferencia de los textos instructivos, estos no hacen énfasis en la secuencia ya que no es un elemento esencial para la organización del discurso.

En la categoría de relatos autobiográficos, los textos se caracterizan por el uso de la narración como principal mecanismo retórico y por tener una historia de vida que da origen al texto. Este es el caso de Juggling Motherhood, en el que la autora, haciendo uso de la metáfora de los malabares, habla de los retos y dificultades que ha enfrentado al asumir el rol de madre siendo estudiante.

Otro relato autobiográfico que se encuentr en el corpus se centra en la experiencia del autor al trabajar como au pair en Alemania. Su texto se estructura a partir de preguntas clave como ¿qué hace un au-pair? y ¿por qué convertirse en au-pair? e incorpora anécdotas y humor a la narración. Algo interesante de este relato es que pone de relieve la visión estereotipada que se tiene de los programas au-pair al pensar que están dirigidos únicamente a la población femenina, seguramente porque involucran el cuidado de niños.

Un tercer relato autobiográfico que se identificó en esta categoría fue Felices árboles amigos (Happy Tree Friends), escrito por una estudiante que cuenta cómo los árboles de la ciudad han acompañado sus experiencias de infancia y adolescencia, al tiempo que hace una descripción de las especies que existen en el campus universitario haciendo énfasis en su diversidad. La estrategia retórica, usada de manera original y creativa, yuxtapone un discurso científico (datos factuales) y un discurso narrativo (experiencias de vida).

En cuarto lugar se encuentran las reseñas con una estructura mucho más transparente y por lo tanto más fácil de identificar. Como objeto de las mismas los autores escogen: un grupo de aplicaciones para aprender lenguas extranjeras (Apps for Learning Foreign Languages), una obra de teatro (Niños Rata), una novela (Carry on) y dos películas (The Danish Girl y Call me by your Name). En todas ellas se presentan detalladas descripciones, narraciones, una opinión personal y una recomendación. En el caso del artículo sobre las aplicaciones, la autora va un paso más allá y realiza una encuesta a estudiantes para determinar cuáles son las más populares en el aprendizaje de lenguas extranjeras e incluye estadísticas para reportar los resultados.

En quinto lugar se identifican dentro del corpus tres textos literarios: "Vuelve a casa" (Come home), ficción que el autor crea a partir de la pérdida de su abuela. "La vida duele y la muerte solo un poco más" (Life hurts. Death, just a little bit more), cuento en el que muy sutilmente la autora narra la experiencia de una adolescente que tiene un aborto. Y "Espacio en blanco" (Blank space), texto literario de corte experimental en el que la autora describe los síntomas que puede presentar un estudiante afectado por una "enfermedad" que inmoviliza a quien la padece, cuando intenta escribir. 
Por último, se encuentra en el corpus un reporte de investigación, el cual, aunque no aborda un tema académico en términos que sean relevantes para la identidad profesional de los estudiantes, sí hace uso de mecanismos retóricos y de estrategias propias de este género. El autor de Sex or not sex comienza por plantearse la pregunta: ¿Es cierto que los estudiantes tienen prácticas sexuales dentro del campus universitario? Y para responderla lleva a cabo una encuesta, realiza entrevistas y analiza los datos encontrados. Posteriormente, incluye una reflexión sobre el diseño de su investigación, las limitaciones metodológicas de su ejercicio y los cambios a introducir si tuviera la oportunidad de realizarlo de nuevo. También hace uso de una voz de autoridad que, aunque ficticia (el estudiante inventa un personaje: "el Doctor Firulais" al que le atribuye un discurso científico), reproduce los usos de estas voces en los textos académicos, a través de citas, opiniones expertas y consejos sobre la manera de conducir el estudio.

Hasta aquí el análisis del corpus constituido por los 37 textos escritos por estudiantes para La Langosta Magazine. Con el segundo interrogante, se busca establecer qué géneros consideran los docentes que deben aparecer en una revista digital estudiantil y contrastarlos con los géneros escritos por los estudiantes. Para acceder a esta información, se realizan entrevistas semiestructuradas con siete docentes del programa de licenciatura en lenguas extranjeras en el cual se diseña la revista.

$\mathrm{Al}$ analizar las entrevistas, se encuentra que las visiones docentes van desde considerar exclusivamente "ejercicios de escritura académica, de vocación científica" hasta recomendar una apertura tal que incluya "escritura experimental y exploraciones estéticas". La mayoría menciona géneros académicos (como el ensayo, la reseña, el artículo de investigación, los trabajos de curso y de grado y la sistematización de experiencias). Se incluyen dos géneros literarios (poesía y cuentos cortos) y traducciones (sin especificar si literarias o académicas). En menor medida se hace referencia el artículo de reflexión, las noticias, la semblanza y el comentario (de un poema, por ejemplo).

Los géneros en común entre las producciones de los estudiantes y lo que los docentes esperan encontrar son el ensayo y la reseña. Ahora bien, la mayor parte de los ensayos identificados en el corpus no son sobre temas propios de la disciplina. Al preguntar a los profesores si consideran pertinentes este tipo de textos para la revista digital estudiantil descrita, sus opiniones están divididas en dos posturas. Algunos están de acuerdo en que no deben tratar temas estrictamente académicos para ser incluidos, lo importante es que este ejercicio "les ofrece la oportunidad de acercarse a los procesos de escritura y de construirse como autores" (O. Vergara, comunicación personal, agosto 17, 2019). Los demás tienen una visión más restrictiva y consideran que "el ensayo debe hacer parte de lo disciplinar para que los profesores o la unidad pueda brindar apoyo a los estudiantes en su iniciativa" (H. Areiza, comunicación personal, agosto 22, 2019).

En el escenario de las reseñas coinciden la mirada docente y la estudiantil: se reseña un libro, una película, un producto o una obra de teatro. La secuencia descripción-narraciónopinión es el guion esperado y seguido. Por su parte, los textos instructivos y los consejos no son mencionados por ninguno de los docentes entrevistados. Este fenómeno llama la atención porque es el segundo texto más común identificado en el corpus de las producciones de los estudiantes. Asimismo, los relatos autobiográficos tienen un lugar significativo en la producción de los estudiantes, sin embargo no aparecen en el discurso docente. Lo 
más cercano que se encuentra es la sistematización de experiencias, pero en este caso los docentes se refieren por ejemplo a la experiencia de los estudiantes en su práctica docente o como aprendices de lenguas extranjeras. Los textos de los estudiantes abordan temáticas que no están estrictamente relacionadas con su identidad profesional.

El reporte de investigación es clasificado como tal porque hace uso de estrategias propias del género, aun cuando el tema no sea de contenido disciplinar propiamente inscrito en el campo de las lenguas extranjeras. Sin embargo, la hipótesis que aquí se plantea es que los docentes entrevistados no lo incluirían dentro de esta categoría porque no trata un tema reconocido como relevante para el contexto disciplinar, sino más relacionado con las vivencias de los estudiantes, en este caso desde su dimensión de jóvenes que se hacen preguntas sobre su vida sexual.

Los temas seleccioandos por los estudiantes revelan dimensiones de su identidad que no siempre son reconocidas por los docentes universitarios. Además de ser estudiantes de Licenciatura en Lenguas Extranjeras en una universidad pública, estos jóvenes tienen una ciudadanía (que es colombiana en este caso), pueden votar, están viviendo el posacuerdo con las FARC (Fuerzas Armadas Revolucionarias de Colombia) en Colombia y en general son de clase media. Además de los temas académicos relevantes para su pertenencia a una comunidad discursiva, también les interesa hablar de relaciones amorosas, de salud sexual y reproductiva, de política migratoria, de ecología, de religión.

Los conceptos de autoría y audiencia se superponen: la revista es creada por estudiantes para estudiantes. Ese otro, es otro yo, por así decirlo, otro yo en el sentido de que es un joven, estudiante, que sigue la misma carrera, o que se hace las mismas preguntas. Hay una cercanía en términos generacionales y en términos del contexto social e histórico, se comparten temas y modos de comunicación; el estudiante selecciona un tema para otro estudiante, colega suyo y par suyo, proyecta de alguna forma su identidad en el otro.

La maestra Silvia Rivera Cusicanqui considera que "todo estudiante es un autor en potencia" (Cusicanqui, 2017), afirmación que implica para los docentes no solo motivar la escritura, sino también llevar a los estudiantes a publicar y aspirar a ser reconocidos como autores. Este reconocimiento atraviesa indiscutiblemente la identidad, la agencia. Por esta razón es fundamental dar un lugar en la academia a las experiencias personales, los relatos autobiográficos, las historias de vida como puntos de partida legítimos no solo para la búsqueda de temas de escritura, sino también para el uso efectivo de estrategias retóricas y para la comprensión de lo que significa construir autoría y audiencia.

\section{Conclusiones}

Las revistas estudiantiles digitales ofrecen oportunidades para la construcción de autoría, a través de la experimentación con distintos géneros y una determinada audiencia; igualmente privilegian la lectura crítica entre pares (y por consiguiente, la comprensión de lo que significa ser miembro de una comunidad de aprendizaje) y el desarrollo de habilidades de escritura en plataformas tecnológicas de comunicación contemporáneas, necesarias para el ejercicio de agencia y participación en múltiples escenarios de la vida.

Las tecnologías digitales ofrecen oportunidades de aprendizaje con respecto al desarrollo de la autoría tales como: el reconocimiento de las múltiples posibilidades de circulación 
de la información y de los modos de comunicación, la apropiación y transformación del conocimiento y la interacción con la audiencia.

Las visiones estudiantiles y las visiones docentes sobre qué tipo de textos son pertinentes para publicaciones universitarias puede diferir y generar falsas expectativas de una $\mathrm{u}$ otra parte cuando se acomete la tarea de crear una revista estudiantil digital. Procede, por ello, tener en cuenta las percepciones de todos los actores para construir acuerdos que garanticen proyectos de largo aliento con apoyo institucional y sentido de pertenencia para todos.

¿Qué hacer con los temas que no son académicos pero que interesan a los estudiantes? Aún cuando los textos no estén estrictamente relacionados con su identidad profesional, en estas prácticas de escritura se desarrollan habilidades que podrán extrapolarse al campo disciplinar. Por esta razón, es un deber docente acompañar a los estudiantes en la escritura de los temas que les interesan y guiarlos en la exploración de los géneros, los modos y las plataformas de comunicación más apropiados para su difusión. Esta es una oportunidad para el aprendizaje de una escritura donde se pongan en escena las múltiples facetas de su identidad en contextos académicos para legitimar dichas facetas y les den un lugar relevante en las prácticas de escritura que se promueven en las aulas de clase.

Las revistas estudiantiles digitales pueden funcionar como laboratorios en los que la labor docente sea la de curar, guiar, editar, sugerir unas formas y, sobre todo, hilar pensamiento paciente y amorosamente junto con los estudiantes, como una forma de construir autoría y audiencia.

\section{REFERENCIAS}

Bajtín, M. (1982). Estética de la creación verbal. México, D.F.: Siglo 21.

Barnechea, M. y Morgan, M. (2010). La sistematización de experiencias: producción de conocimientos desde y para la práctica. Tendencias y Retos, (15), 97-107. Disponible en http://www.ts.ucr.ac.cr/binarios/tendencias/rev-co-tendencias-15-07.pdf

Bazerman, Ch. (2009). Genre and Cognitive Development: Beyond Writing to Learn. Pratiques, (143-144), 127-138. https://doi.org/10.4000/pratiques.1419

Blanco, M. y Mosquera, H. (2019). El lenguaje valorativo en artículos de investigación sobre interculturalidad en la enseñanza de lenguas: Análisis discursivo de las introducciones de artículos publicados en una revista internacional especializada en lenguaje y comunicación intercultural. (Tesis de Maestría). Universidad del Valle, Cali, Colombia.

Carlino, P. (2005). Escribir, leer y aprender en la universidad: una introducción a alfabetización académica. (1 ed.). Buenos Aires: Fondo de Cultura Económica.

Carvajal, G. (2008). Lecturas y escrituras juveniles: entre el placer, el conformismo y la desobediencia. Cali: Editorial Universidad del Valle.

Cusicanqui, S. (Junio, 2017). Sociología de la imagen: pensar con los pies en la tierra. En, ColectivxChixi, Cátedra Libre "Ideas Menores", Universidad del Valle, Cali, Colombia.

Flórez, E. (2018). Currículo oculto. En, Mincultura, Reconocimientos a la crítica y el ensayo: Arte en Colombia, versión 13 (pp. 36-40). Bogotá, D.C.: Ediciones Uniandes.

Foucault, M. (1969). Qu'est-ce qu'un auteur? Bulletin de la société francaise de philosophie, 63(3), 73-104. Disponible en http://www.sofrphilo.fr/quest-ce-quun-auteur/ 
Gee, J. (2013). Good Video Games and Good Learning. Collected Essays on Video Games, Learning and Literacy. N.Y.: Peter Lang Publishing.

Hobbs, R. y Smith, L. (2018). Digital Authorship: Issues and Implications for Educators. Primer, 46(2), 7-17. Disponible en https://mediaeducationlab.com/sites/default/files/ Digital\%20Authorship\%20Primer\%20Hobbs\%20and\%20Smith\%202018.pdf

Hyland, K. (2006). The 'other' English: Thoughts on EAP and Academic Writing. The European English Messenger, 15(2), 34-47. Disponible en https://essenglish.org/messenger/wp-content/uploads/sites/2/2016/01/152-34-57.pdf

Jara, O. (abril, 2001). Dilemas y desafíos de la sistematización de experiencias. En, Intercooperation, Agricultura sostenible campesina de montaña, Conferencia llevada a cabo en Seminario ASOCAM, Cochabamba, Bolivia. Disponible en https://www.grupochorlavi.org/webchorlavi/sistematizacion/oscarjara.PDF

Lankshear, C. y Knobel M. (2008). Digital literacy. Concepts, policies and practices. N.Y.: Peter Lang Publishing. Disponible en https://www.academia.edu/293040/Digital_Literacies_Concepts_Policies_and_Practices

Lillis, T. (2003). Student Writing as 'Academic Literacies': Drawing on Bakhtin to Move from Critique to Design. Language and Education, 17(3), 192-207. https://doi. org/10.1080/09500780308666848

Mannion, G., Ivanič, R. \& LfLFE. (2007). Mapping literacy practices: theory, methodology, methods. International Journal of Qualitative Studies in Education, 20(1), 15-30. https://doi.org/10.1080/09518390600924063

Medlock, C., Spires, H. y Kerkhoff, S. (2017). Digital Literacy for the 21st Century. In: M. Khosrow-Pour, Encyclopedia of Information Science and Technology (Chapter 194). (pp. 2235-2242). Hershey: IRMA. https://doi.org/10.4018/978-1-5225-2255-3.ch194

Miller, C. (1994). Rhetorical Community: The Cultural Basis of Genre. En: A. Freedman and P. Medway (eds), Genre and the new rhetoric (pp. 67-78). London: Taylor \& Francis.

Miller, C. \& Kelly, A. (2016). Discourse Genres. In: A. Rocci and L. Saussure (eds.), Handbooks of Communication Science [HoCS], Vol. 3 (pp. 269-286). Berlín: De Gruyter Mouton. https://doi.org/10.1515/9783110255478-015

Netto, A. M. (2007). Do modelo ao estilo: possibilidades de autoria em contextos acadêmico-científicos. En, E. Calil, (org.), Trilhas da escrita: autoria, leitura e ensino (pp. 171-207). São Paulo: Cortez.

Polyzou, A. (2008). Genre-based data selection and classification for critical discourse analysis. En, M. KhosraviNik and A. Polyzou (eds.), Papers from the Lancaster University Postgraduate Conference in Linguistics \& Language Teaching (LAEL PG). vol. 2, 104-135. Lancaster: Lancaster University. Recuperado de https://eprints.lancs.ac.uk/ id/eprint/1877

Selener, D., Purdy, Ch. y Zapata, G. (1996). Manual de sistematización participativa: Documentando, evaluando y aprendiendo de nuestros proyectos de desarrollo. Quito: Abya-Yala. Recuperado de https://digitalrepository.unm.edu/cgi/viewcontent. cgi?referer=https://www.google.com/\&httpsredir=1\&article=1505\&context=abya_yala

Sheedlo, S. (1998). Finding Reasons to Publish Student Writers. Language Arts Journal of Michigan: 14(2), 47-50. https://doi.org/10.9707/2168-149X.1417 
Urra, M., y Pérez, A. (2015). El Premio Rubén Ardila a la Investigación Científica en Psicología (2005-2015): De la Psicología Organizada a la Historia de las Ciencias del Comportamiento. Cultura Educación y Sociedad, 6(2), 77-89. Recuperado de https:// revistascientificas.cuc.edu.co/culturaeducacionysociedad/article/view/1042

Zainuddin, H. y Moore, R. A. (2003). Audience Awareness in L1 and L2 Composing of Bilingual Writers. TESL-EJ, 7(1). Disponible en http://tesl-ej.org/ej25/a2.html 